\title{
Variability of Door-to-Device Times at a Rural Tertiary Care Center
}

Victor A. Abrich, MD; Roxann Rokey, MD; Satya S.V. Bhupathi, MD, MPH; and Juan E. Mesa, MD

Objectives: Target door-to-device (DTD) time for ST-elevation myocardial infarction (STEMI) patients has been 90 minutes, with no distinction between urban and rural hospitals. Rural hospitals have longer DTD times for transferred patients attributed to long transportation times from referring hospitals. Longer DTD times have also been reported during after-hours. The aim of the study was to determine whether DTD times at our rural facility were impacted by arrival method, arrival time period, and season.

Design: Retrospective chart review.

Setting: Rural tertiary care center in central Wisconsin.

Methods: We studied 4I 2 patients presenting with STEMI after initiation of the Rescue One program for rapid triage and transfer from October 2006 through December 2012. They were subdivided by arrival method, arrival time (ON=Monday-Friday, 8 AM-5 PM; OFF=after-hours, weekends, holidays), and season. Median DTD times and proportions below and above 90 minutes were compared.

Results: Median DTD time for all groups, which include both directly admitted and transferred patients, was 85 minutes with $60 \%$ of patients achieving DTD times below 90 minutes while 30-day mortality was 5.3\%. Median DTD time was 67 minutes for the Emergency Department (ED) $(n=164)$, 95 minutes for Transfers $(n=204), 68$ minutes for Urgent Care $(n=22)$ and 86 minutes for Field $(n=22)$. ED had the highest proportion of patients achieving goal DTD time (81\%) compared to Transfers (42\%). Patients arriving by ED during OFF hours had a median DTD time 28 minutes longer than during $\mathrm{ON}$ hours with $21 \%$ fewer patients achieving goal DTD time, attributed to the time required to call in the catheterization team. Seasonal variability was observed due to differences in pre-hospital ambulance transportation times in the Field group.

Conclusions: Our data confirm that in a rural facility such as ours, ED patients arriving during afterhours and transferred patients have longer DTD times. Methods are being implemented to shorten the time to assemble the catheterization lab team during after-hours. Better performance will be seen once the first medical contact to device (FTD) time goal of 120 minutes for transferred patients is adopted at our institution. Fibrinolytic therapy should be considered at referring institutions where the FTD time is expected to exceed I 20 minutes.

Keywords: Door-to-device time; ST-elevation myocardial infarction; Seasonal variability/variation;

Time period/arrival time 
he current best reperfusion strategy for patients presenting with an ST-elevation myocardial infarction (STEMI) is primary percutaneous coronary intervention (PCI). ${ }^{1}$ The American College of Cardiology (ACC) and American Heart Association (AHA) set a goal to attain a door-to-device (DTD) time below 90 minutes for patients with STEMI. This included patients who initially presented to facilities not equipped to perform PCI and were then transferred to hospitals that had this capability. Hospitals nationwide have managed to dramatically shorten their DTD times since the target of 90 minutes was introduced in 1999. Unfortunately, delays in inter-hospital transfer have been responsible for failing to reach this target for the majority of the transfer patient subset.

Target DTD time is identical for both urban and rural hospitals. A hospital is considered rural if it is located in an area of low population density, compared to an urban or suburban hospital. To be considered a rural tertiary care center, the hospital must meet additional criteria outlined by the Centers for Medicare \& Medicaid Services. The first criterion is that the hospital must have at least 275 beds. If this does not apply, criteria for the Medicare patient population served at the hospital may be applied and includes stipulations that at least $50 \%$ of patients must be referred from other hospitals, $60 \%$ of patients must live at least 25 miles away, and $60 \%$ of all Medicare services must be provided to patients living at least 25 miles away. ${ }^{2}$

Longer DTD times for transferred patients were reported for rural teaching hospitals compared to urban centers. ${ }^{3}$ This disparity is partially attributable to greater geographic separation of facilities leading to relatively long transportation times. In the rural setting, other factors posited for contributing to transfer delays include inadequate staffing of emergency medical services personnel, lack of 24/7 in-house cardiac catheterization laboratory team, effects of weather on transportation, and lack of experience managing STEMI at community hospitals due to relatively infrequent occurrence. ${ }^{4}$ Nevertheless, feasibility of achieving DTD times of $<90$ minutes in rural settings has been demonstrated, both with and without a protocol for rapid triage and transfer. ${ }^{5,6}$

The time of day a patient presents with STEMI is an important prognostic factor. In a recent study looking at the time of presentation for STEMI patients, prolonged DTD times were documented at higher frequency during after-hours compared to regular business hours. This meta-analysis reported a small increase in short-term mortality compared to regular business hours. ${ }^{7}$ The aim of the current study was to determine whether any differences exist in DTD times at our rural tertiary care center between regular business hours and after-hours, including weekends and holidays. We also examined seasonal variability for DTD times and impact on transportation time to the hospital, which have not been studied previously.

\section{Methods}

St. Joseph's Hospital (SJH), a Ministry Health Care hospital, is a 504-bed rural tertiary care center located in Central Wisconsin. It is one of the largest hospitals in Wisconsin and is the only major rural referral center in the state. It serves a predominantly rural service area encompassing central and northern Wisconsin, serving 19,000 inpatients and 38,000 outpatients annually. SJH has the capability of performing PCI, receiving STEMI referrals from 17 different hospitals located throughout central and northern Wisconsin. Patients are transported by ambulance, helicopter, and ambulancehelicopter intercept with distances reaching up to 98 miles. $\mathrm{SJH}$ is partnered with and physically adjacent to Marshfield Clinic (MC) with whom it shares a combined electronic medical record and from which a number of STEMI patients are referred through its Urgent Care department.

The Rescue One program for rapid triage and transfer of STEMI patients within a regional network of referring community hospitals was initiated in October 2006 in an effort to improve DTD times. The team consists of the on-call cardiologist, the interventional cardiologist, and catheterization laboratory nurses and technicians, who are in-house only during regular business hours. During after-hours, including holidays and weekends, the Rescue One team is called in to the catheterization laboratory with the goal of being ready within 30 minutes of being notified of an emergent STEMI case. Any physician at an originating hospital can call a "Rescue One" and activate the STEMI protocol before the electrocardiogram is reviewed by a cardiologist. This includes physicians working in the Emergency Department and Urgent Care. Emergency Medical Services can also electronically transmit electrocardiograms from the field to the hospital, at which point the emergency department physician can activate the STEMI protocol before the patient arrives.

Since initiation of the Rescue One program, 420 patients presented with STEMI at our institution between October 2006 and December 2012. There were 8 patients excluded: 3 who were later diagnosed as having non-STEMI, 1 transferred patient who had received thrombolytics, and 4 transferred patients who initially had non-diagnostic electrocardiograms at referring hospitals but later had diagnostic electrocardiograms upon arrival to our Emergency Department (figure 1). After these exclusions, 412 patients remained. In-house mortality data and DTD times were collected retrospectively from the SJH Cardiac Database Registry that participates in the American College of Cardiology-National Cardiovascular Data Registry (ACC-NCDR) database, while 30-day mortality data were collected from the combined electronic medical record for $\mathrm{MC} / \mathrm{SJH}$. We used this data to perform a retrospective observational study in this select cohort of patients with STEMI. This study was approved by the Marshfield Clinic Research Foundation Institutional Review Board that serves MC and SJH. 


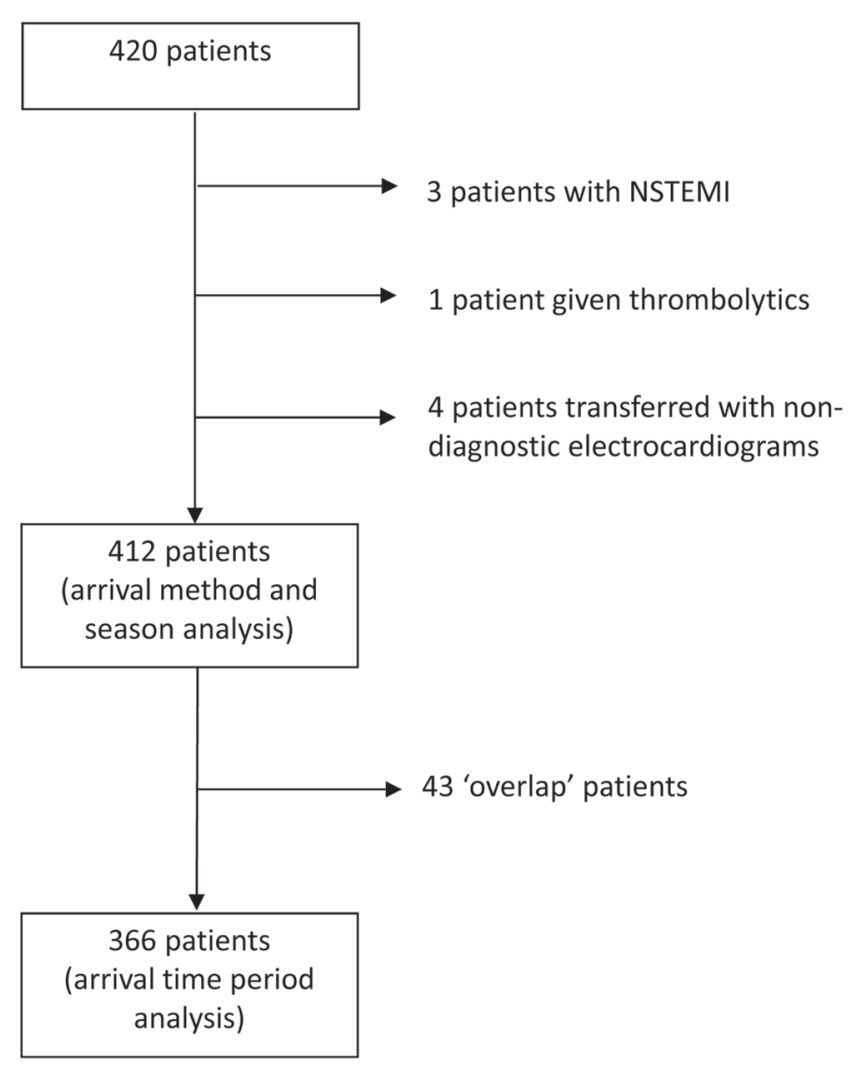

Figure 1. Flow chart showing patient exclusions for different DTD time analyses.

DTD time was defined as the time from arrival to the facility to time of catheterization device deployment in the affected coronary artery. At our institution, the DTD time was applied to patients presenting by private vehicle directly to the Emergency Department or to Urgent Care. In some cases, patients who arrived at Urgent Care were transferred to the Emergency Department before going to the catheterization laboratory due to time of presentation and the need for telemetric monitoring. For patients arriving at $\mathrm{SJH}$ from the field via ambulance, the first medical contact to device (FTD) time was used as a surrogate measurement; this started from the time emergency medical services made contact with the patient after arriving on scene, continuing through with transportation time, and ending at the time the device was deployed. The door-1-to-device (D1TD) time was used as a surrogate measurement for transferred patients, starting from arrival to, and departure from, the referring hospital (door-indoor-out [DIDO] time), continuing with transportation time, and ending with the time of device deployment. Whereas the initial electrocardiogram on presentation was non-diagnostic for some patients presenting to our emergency department and outside hospitals $(\mathrm{n}=29)$, a subsequent diagnostic electrocardiogram prompted activation of the Rescue One protocol. In these cases, electrocardiogram-to-device time was used as a surrogate for DTD time, and was defined as the time from when a STEMI was diagnosed by electrocardiogram to the time of device deployment. Catheterization laboratory-to-device (CLD) time was defined as the interval beginning with arrival to the catheterization laboratory to device deployment.

The DTD times were subset into seasonal quartiles, defined by the following intervals: spring (March 20 to June 20), summer (June 21 to September 21), fall (September 22 to December 20), and winter (December 21 to March 19). The DTD times were further subset to time ranges representing ON hours (Monday through Friday, 8:00 AM to 5:00 PM); OFF hours (Monday through Friday, 5:01 PM to 7:59 AM as well as weekends and statutory holidays); and 'overlap' (patients with DTD times that happened to occur during the transition between ON and OFF hours). Finally, patients were also classified by 1 of 4 methods of arrival: Emergency Department, Urgent Care, Transfer, and Field.

Median DTD times and interquartile ranges were calculated for each of the seasons and methods of arrival. For arrival time, median DTD times and interquartile ranges were calculated for the ON and OFF hours subsets; DTD times in the 'overlap' subset $(n=43)$ were excluded from arrival time analysis, but were included in analyses of seasons and method of arrival (figure 1). Non-parametric statistical tests were then

Table 1. Median DTD times and proportional distribution by method of arrival

\begin{tabular}{llllll}
\hline & $\begin{array}{l}\text { Emergency } \\
\text { Department } \\
(\mathbf{n = 1 6 4 )}\end{array}$ & $\begin{array}{l}\text { Urgent Care } \\
(\mathbf{n = 2 2})\end{array}$ & $\begin{array}{l}\text { Field } \\
(\mathbf{n = 2 2})\end{array}$ & $\begin{array}{l}\text { Transfers } \\
(\mathbf{n = 2 0 4 )}\end{array}$ & $\boldsymbol{P}$ value \\
\hline Median DTD time (minutes) & $67[51,84]$ & $68[54,98]$ & $86[62,100]$ & $95[82,112]$ & $<0.0001^{\dagger}$ \\
$\mathrm{n}(\%) \leq 90$ minutes & $133(81 \%)$ & $16(72 \%)$ & $13(59 \%)$ & $86(42 \%)$ & $<0.0001^{\ddagger}$ \\
$\mathrm{n}(\%)>90$ minutes & $31(19 \%)$ & $6(27 \%)$ & $9(41 \%)$ & $118(58 \%)$ & \\
$\mathrm{n}(\%) \leq 120$ minutes & & & & $166(81 \%)$ & $\mathrm{N} / \mathrm{A}$ \\
$\mathrm{n}(\%)>120$ minutes & 38 & 17 & 11 & $38(19 \%)$ & $\mathrm{N} / \mathrm{A}$ \\
$\mathrm{n} \mathrm{ON}$ & 112 & 2 & 8 & 117 & \\
$\mathrm{n}$ OFF & & & & & \\
\hline
\end{tabular}

DTD, Door-to-device.

*Interquartile ranges are displayed in square brackets next to median door-to-device time.

†Kruskal-Wallis test used to calculate $P$ value.

$\neq$ Chi-Square test used to calculate $P$ value. 


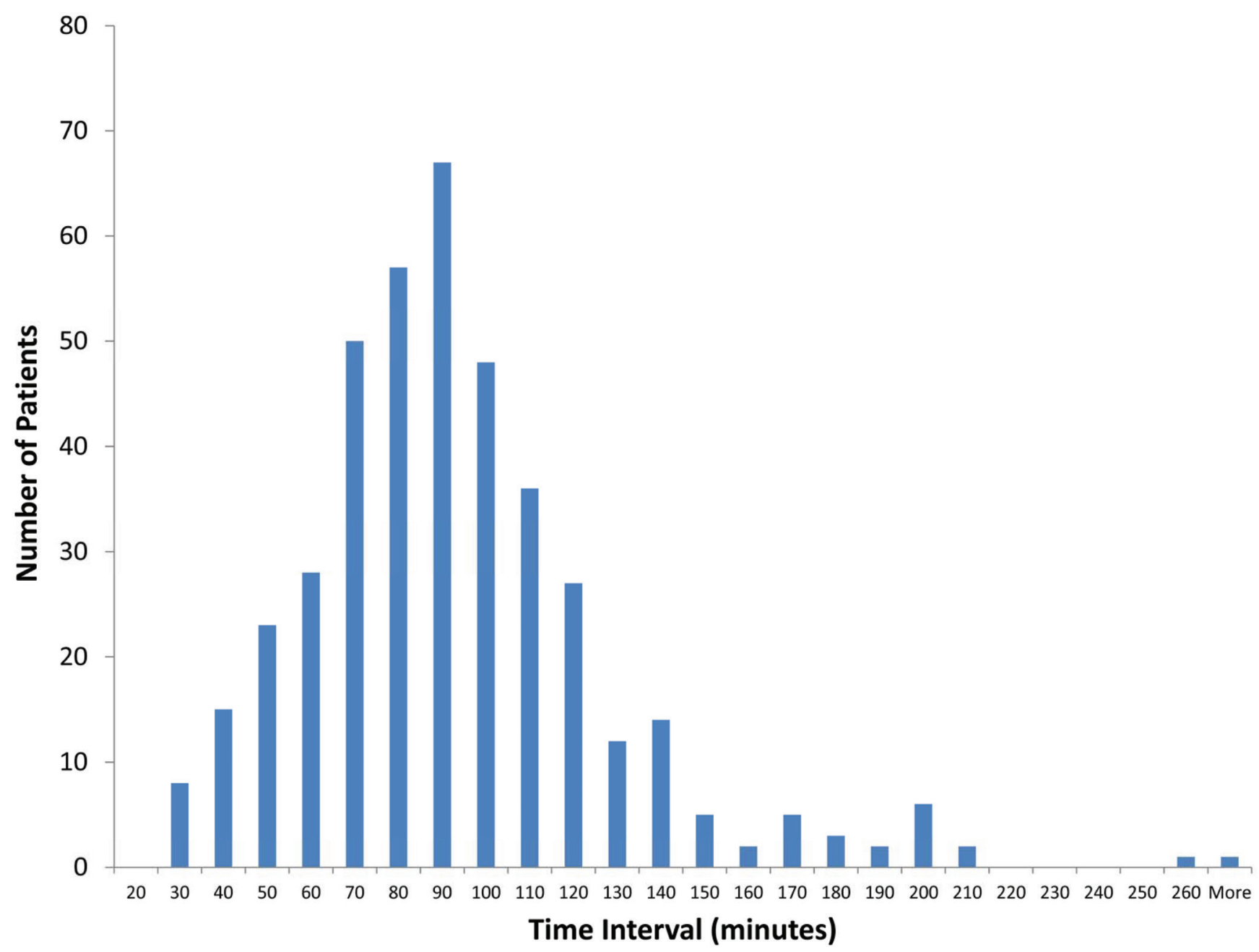

Figure 2. DTD time histogram showing a positive skew. Patients are separated by 10 minute intervals.

applied to the DTD times using JMP 10 Statistical Discovery software from SAS (Cary, NC). The Wilcoxon Rank Sums test for comparison of medians from two groups was used to compare median DTD times for ON vs. OFF hours, while the Kruskal-Wallis test for comparison of medians in more than two groups was used to compare median DTD times by method of arrival and across seasons. The proportions of patients with DTD times below and above the goal DTD time of 90 minutes were compared by method of arrival, arrival time period, and season. The $P$ values for method of arrival and seasons were calculated using the Chi-Square test, while the $P$ values for time period were calculated using Fisher's Exact test. A test was considered statistically significant if the $P$ value was calculated to be $<0.05$.

\section{Results}

In this study, DTD times were found to have a positively skewed distribution (figure 2). The median DTD time for all groups, which included both directly admitted and transferred patients, was 85 minutes, with $60 \%$ of patients achieving DTD times below 90 minutes. Of the 412 patients included in this study, 8 patients died in the hospital (in-house mortality $1.9 \%$ ) while 22 patients died within 30 days (30-day mortality
$5.3 \%$ ). There was no association demonstrated between mortality and DTD times below 90 minutes $(P=1.000)$. Observed-to-expected mortality ratios for each calendar year during the study period from 2007 to 2012 were $1.18,0.93$, $0.65,0.78,0.88$, and 0.27 , respectively.

Median DTD times and the proportions of patients with DTD times above and below 90 minutes were compared among the four methods of arrival (table 1). Differences between DTD times were found to be statistically significant $(P<0.0001)$. No differences were observed between the median DTD times for the Urgent Care and Field groups on head-to-head comparison; this is likely because such differences were too small to be detected in these small sample sizes. The majority of patients in the Emergency Department, Urgent Care, and Field groups achieved DTD times below 90 minutes, which was not seen in the Transfers group. However, $81 \%$ of transferred patients achieved D1TD times below 120 minutes.

The distribution of time was examined for each of the two most common methods of arrival, Emergency Department and Transfers. Median DTD time for the Emergency Department group was found to be 28 minutes shorter during 


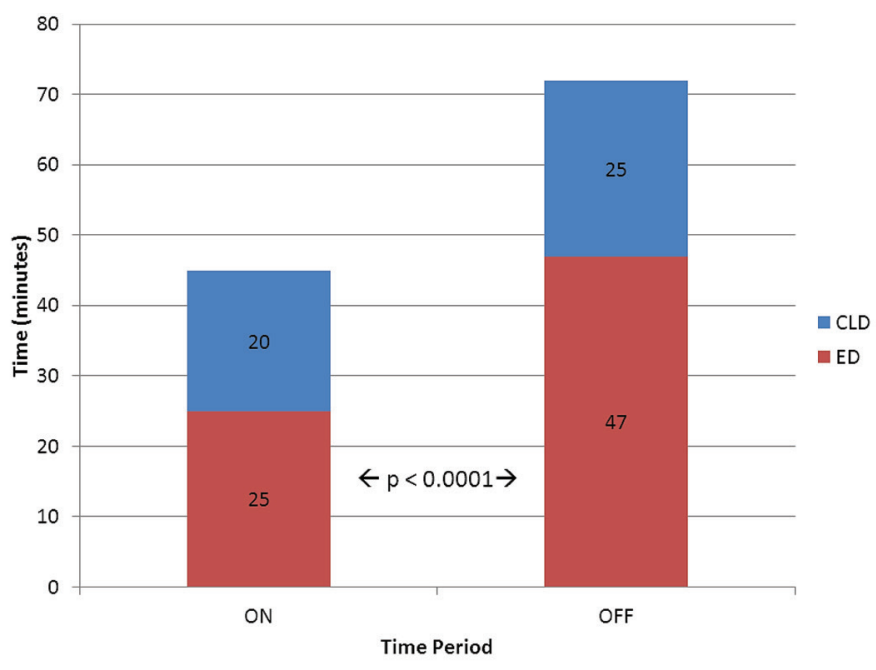

Figure 3. Time distribution of median DTD time for patients arriving via the Emergency Department (ED). Time spent in emergency department was shorter during ON hours compared to OFF hours $(P<0.0001)$. [ED = time spent in ED, CLD = catheterization laboratory-to-device time].

ON hours than during OFF hours $(P<0.0001)$, while no difference across time period was observed for the Transfers group. The overall DTD time for the Emergency Department group consisted of the time spent in the emergency department and the CLD time (figure 3). The median time spent in the emergency department was found to be significantly longer during OFF hours than during ON hours $(P<0.0001)$, while the median CLD time showed no significant difference across $\mathrm{ON}$ and OFF arrival time periods. For transferred patients, the D1TD time consisted of the DIDO time, the transportation time, and the CLD time (figure 4). Whereas no significant differences in median DIDO time and median transportation time were noted across the time period for patients transferred from outside hospitals, longer median CLD times were observed during OFF hours compared to ON hours $(P=0.0146)$. In addition, 85 transferred patients $(58 \%)$ had DIDO times that exceeded 30 minutes.

The proportions of patients with DTD times below and above 90 minutes were examined across $\mathrm{ON}$ and OFF arrival time periods for the Emergency Department and Transfers methods of arrival (table 2). For patients presenting to the emergency department, 21\% fewer patients achieved goal DTD times during OFF hours compared to ON hours $(P=0.0030)$. For

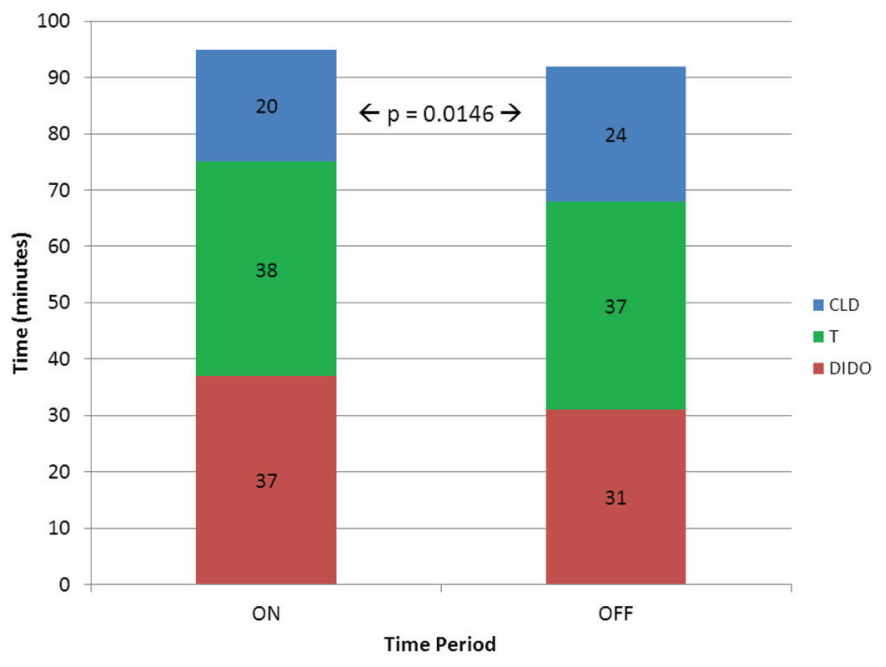

Figure 4. Time distribution of median D1TD time for patients transferred from outside facilities. Catheterization lab to device time was shorter during ON hours compared to OFF hours ( $P$ $=0.0146$ ) [DIDO = door-in-door-out time, $\mathrm{T}=$ transportation time, CLD = catheterization laboratory-to-device time.].

patients being transferred from outside facilities, no significant difference in the proportion of patients with DTD times below 90 minutes across the $\mathrm{ON}$ and OFF arrival time periods was noted $(P=0.3251)$.

Statistically significant differences between median DTD times across seasons (table 3$)$ were detected $(P=0.0254)$; however, subgroup analysis by arrival time period did not reveal any significant difference across seasons during ON hours or OFF hours. This statistical significance disappeared when the DTD times from the Field group $(\mathrm{n}=22)$ were removed from the analysis $(P=0.1430)$. Subgroup analysis of the Field group revealed statistically significant differences across seasons for median DTD times $(P=0.0134)$ and median transportation times $(P=0.0379)$. In this small subset of patients, individual transportation times for all patients in the Field group were plotted by season (figure 5). Median transportation times were shortest in the fall (44 minutes, $\mathrm{n}=10$ ) and longest in the spring (74 minutes, $\mathrm{n}=5$ ). The proportion of patients with DTD times below 90 minutes ranged from $51 \%$ to $69 \%$ in spring and fall, respectively, though these differences were not found to be statistically significant $(P=0.0541)$.

Table 2. DTD time proportions during ON vs. OFF hours for Emergency Department and Transfers groups

\begin{tabular}{|c|c|c|c|c|}
\hline & \multicolumn{2}{|c|}{ Emergency Department } & \multicolumn{2}{|c|}{ Transfers } \\
\hline & $\mathrm{n}(\%) \leq 90 \mathrm{~min}$ & $\mathrm{n}(\%)>90 \mathrm{~min}$ & $\mathrm{n}(\%) \leq 90 \mathrm{~min}$ & $\mathrm{n}(\%)>90 \mathrm{~min}$ \\
\hline ON & 37 (97\%) & $1(3 \%)$ & $23(36 \%)$ & $41(64 \%)$ \\
\hline OFF & $85(76 \%)$ & $27(24 \%)$ & $50(43 \%)$ & $65(57 \%)$ \\
\hline$P$ value* & \multicolumn{2}{|c|}{0.0030} & \multicolumn{2}{|c|}{0.3251} \\
\hline
\end{tabular}

*Fisher's exact test used to calculate $P$ values.

DTD=door-to-device 


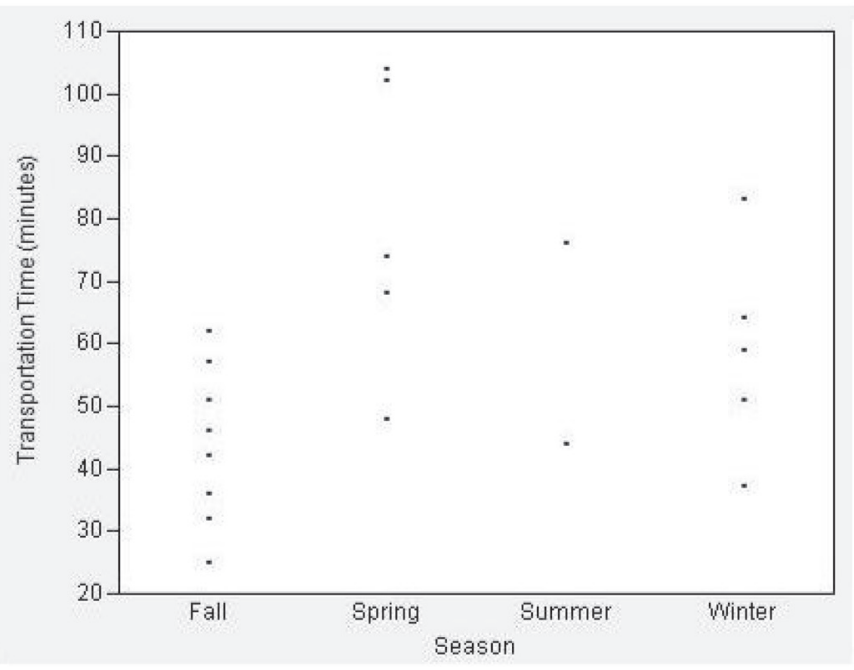

Figure 5. Individual transportation times of patients in the Field group by season. Image generated using JMP 10 Statistical Discovery from SAS (Cary, NC).

Performance of the Rescue One program was evaluated over time for the two largest groups, Emergency Department and Transfers. The median DTD times for the Emergency department from 2007-2012 are all under 90 minutes. Over the course of the study, there was an improvement in yearly median DTD time from 82 minutes in 2008 to 54 minutes in 2012. This is a significant improvement over the median DTD time of 107 minutes in 2004, before implementation of the Rescue One program. The Transfers group also showed a significant improvement in their yearly D1TD times during the study period. Median D1TD time was highest at 108 minutes in 2009 and improved to 89 minutes by 2012 .

\section{Discussion}

In the present study, statistically significant differences in DTD times by method of arrival were observed $(P<0.0001)$. Median DTD times were found to be shortest in the Emergency Department and Urgent Care groups, confirming that patients who present directly to the hospital have the shortest DTD times. The longer DTD times in the Field and Transfers groups can be explained by the transportation time, which was included in the surrogate DTD time measurements for both of these methods of arrival (FTD time and D1TD time, respectively). This added transportation time is a strong contributing factor to why fewer patients achieve DTD times below 90 minutes for these two methods of arrival. Fortunately, transferred patients will have an increase in the target time to receive PCI, since the 2013 ACC/AHA guidelines recommend a FTD time of less than 120 minutes for transferred patients. ${ }^{1}$ In our study, $81 \%$ of transfers had D1TD times below 120 minutes, compared to $42 \%$ with D1TD times below 90 minutes. The D1TD time is an underestimation of the FTD time, since it does not take into account ambulance transportation time to the referring hospital. Although this transportation time may account for part of the additional 30 minutes added to the target time, this extra time may enable more transferred patients to achieve this new goal once this measurement is adopted for national reporting.

Statistically significant differences in DTD time during ON/ OFF arrival time periods were observed with overall median DTD times being longer during OFF hours than ON hours ( $P=0.0197)$, a finding consistent with other studies. ${ }^{7}$ Subgroup analysis of the two most common methods of arrival found this difference between ON and OFF hours to be present only in the Emergency Department group, where the median DTD time was 28 minutes longer during OFF hours than during ON hours $(P<0.0001)$. Physician coverage in the emergency department was lowest during third shift (11:00 PM to 7:00 $\mathrm{AM}$ ); however no difference was observed between $\mathrm{ON}$ and OFF hours with respect to time to activation of the STEMI protocol, which includes the time from patient arrival to acquisition of the electrocardiogram. Patients were spending a median time of 22 minutes longer waiting in the emergency department during OFF hours than during ON hours $(P<0.0001)$ before being transferred to the catheterization laboratory. This wait time can be attributed to the time required to assemble the catheterization laboratory team during OFF hours, with a goal of 30 minutes set at our institution. This difference resulted in $21 \%$ fewer patients achieving DTD times of under 90 minutes during OFF hours compared to ON hours $(P=0.0030)$.

For transferred patients, no difference in overall median D1TD time was observed between ON and OFF hours. This is because after being called in during OFF hours, the catheterization laboratory team has enough time to assemble

Table 3. Median DTD times and proportional distribution across seasons.

\begin{tabular}{llllll}
\hline & $\begin{array}{l}\text { Spring } \\
(\mathbf{n = 1 0 8 )}\end{array}$ & $\begin{array}{l}\text { Summer } \\
(\mathbf{n = 9 8 )}\end{array}$ & $\begin{array}{l}\text { Fall } \\
(\mathbf{n = 1 1 3})\end{array}$ & $\begin{array}{l}\text { Winter } \\
(\mathbf{n = 9 3 )}\end{array}$ & $\boldsymbol{P}$ value \\
\hline Median DTD time (minutes) & $90[71,112]$ & $84[66,108]$ & $78[64,96]$ & $86[65,102]$ & $0.0254^{\dagger}$ \\
ON & $77[59,112]$ & $76[55,110]$ & $69[47,91]$ & $88[62,104]$ & $0.1396^{\dagger}$ \\
OFF & $94[80,112]$ & $85[67,112]$ & $83[67,96]$ & $86[70,101]$ & $0.0868^{\dagger}$ \\
n (\%) $\leq 90$ minutes & $55(51 \%)$ & $58(59 \%)$ & $78(69 \%)$ & $57(61 \%)$ & $0.0541^{\ddagger}$ \\
n $(\%)>90$ minutes & $53(49 \%)$ & $40(41 \%)$ & $35(31 \%)$ & $36(39 \%)$ & \\
\hline
\end{tabular}

*Interquartile ranges are displayed in square brackets next to median door-to-device time.

† Kruskal-Wallis test used to calculate $P$ value.

${ }^{\ddagger}$ Chi-Square test used to calculate $P$ value. 
on site before the patient arrives from the referring facility. Analysis of the time distribution for transferred patients did not reveal any statistically significant difference in DIDO time or transportation time across time period, however the median CLD time was found to be 4 minutes longer during OFF hours than during ON hours $(P=0.0146)$. This statistically significant difference was no longer detected after 28 technically difficult cases were removed from the comparison $(P=0.1150)$. These included cases with difficult vascular access and challenging coronary artery lesions as documented by the interventional cardiologist on the case. In addition to technically difficult cases, another possible explanation for this finding may be that patients transferred during OFF hours are not adequately prepped before arrival at the catheterization lab (eg, shaving the groin area), prolonging the CLD time. Nevertheless, the proportion of patients achieving D1TD times under 90 minutes across time period was not affected by this small difference in CLD time $(P=0.3251)$.

Another important finding in transferred patients was that $58 \%$ of them had DIDO times exceeding 30 minutes. The target DIDO time set by the ACC/AHA is 30 minutes or less, as this has been strongly associated with achieving D1TD times below 90 minutes. ${ }^{8}$ Therefore, the DIDO time can be used to guide the choice of reperfusion therapy, either PCI or fibrinolysis. In order to apply the goal DIDO time of 30 minutes to the FTD time goal of 120 minutes, pre-hospital ambulance transportation times to referring institutions need to be considered. When allotting 60 minutes to the sum of the transportation time to the accepting facility and the CLD time, there are 60 minutes that can be utilized before transferring the patient. If the pre-hospital ambulance transportation time plus the DIDO time exceeds 60 minutes, the FTD time is likely to exceed 120 minutes and fibrinolysis should be considered as a better option in the absence of any absolute contraindications.

Variability of DTD times across seasons was observed when all patients were taken into account $(P=0.0254)$. This seasonal variability was found to stem from the Field group and can be attributed to differences in ambulance transportation times between spring and fall. This might be due to differing management strategies of individual ambulance crews upon arrival to the scene. A recent shift in protocol has been made to emphasize a modified "scoop and run" approach, whereby emergency medical services load the patient onto the ambulance upon arrival to the scene for immediate transportation rather than spending time on diagnosis at the scene. An electrocardiogram is obtained en route and transmitted to the nearest emergency department. If a physician reads it as a STEMI, a "Rescue One" is activated. The observed difference may also be due to a sampling error, since pre-hospital transportation times are represented only by the ambulance crews transporting patients in the Field group directly to $\mathrm{SJH}$. It does not take into account transportation times to referring facilities. Although not included in this study, future research could include such transportation times in order to analyze the FTD time for transferred patients. Nevertheless, the proportion of patients achieving target DTD times of 90 minutes or less was not affected by the observed seasonal variability $(P=0.0541)$.

There are a few published studies examining DTD times in rural tertiary care centers of comparable size. A 437-bed tertiary care center in rural central Pennsylvania demonstrated a progressive annual improvement in median DTD times for patients transferred by helicopter and ambulance after initiating a system for rapid triage and transfer of STEMI patients. Overall, 37\% of the collective 568 transferred patients over a 5-year period achieved DTD times of 90 minutes or less with no difference between $\mathrm{ON}$ and $\mathrm{OFF}$ hours. ${ }^{5}$ Beginning from a median DTD time of 189 minutes $(n=109)$ in 2004, they achieved median DTD times of 95 minutes $(n=118)$ in 2007 and 88 minutes $(n=188)$ in 2008, with an in-house mortality of $3.4 \%$.

Other rural hospitals have reported a higher success rate in achieving DTD times below 90 minutes for transferred patients. Two referral hospitals for STEMI patients in central rural Illinois, one with 563 beds and the other with 731 beds, reported $58 \%$ of patients achieving DTD times below 90 minutes with a median DTD time of 117 minutes $(n=230)$ and a 30-day mortality of 3.7\%. ${ }^{9}$ Similarly, a 340-bed hospital with PCI-capability in rural West Virginia that examined mean DTD times for 89 transferred patients, reported that $55 \%$ of them attained DTD times under 90 minutes without a system of rapid triage and transfer in place. ${ }^{6}$ While the aforementioned studies focused on transferred patients, a 396-bed referral hospital for STEMI patients located in rural New Hampshire collectively examined arrival of STEMI patients from both the emergency department and the field. ${ }^{4}$ After initiating a protocol for rapid triage and transfer of STEMI patients, they reported $77 \%$ of patients achieving DTD times below 90 minutes with a median DTD time of 67 minutes $(n=79)$ and a 30 -day mortality of $6.7 \%$.

Compared to the above published studies, our data showed comparable performance. Of the 204 patients in the transfer cohort, $42 \%$ of them achieved DTD times below 90 minutes, with a median DTD time of 95 minutes and no difference between $\mathrm{ON}$ and OFF hours. Better performance was observed in patients arriving from the emergency department and from the field. Of the combined 186 patients from these 2 groups, $78 \%$ of them achieved DTD times below 90 minutes with a median DTD time of 68 minutes.

Our study did not show a demonstrable effect on mortality in association with achieving DTD times below 90 minutes. This is consistent with recent evidence that shorter DTD times have not led to decreased mortality on a national scale. ${ }^{10}$

Although our data are similar to other that of other rural institutions, the main limitation of our study is that it is a small retrospective observational study that includes data 
over a limited time period. We anticipate that with more data collection over a longer period of time, we would generate results with greater statistical power. Another limitation is that technology has substantially changed over the course of the study period. Not all ambulances have the capability of using in-field 12-lead electrocardiograms, especially in smaller rural communities. As adaptation rates increase, we anticipate more standardization of management strategies and shorter FTD times.

In taking a closer look at some of the DTD time surrogates, a few inconsistencies between measurements arise. Although the FTD time for Field patients begins from the time emergency medical services makes first contact with the patient, management from that point onward is inconsistent among emergency medical services crews. The FTD times obtained do not specify whether the emergency medical services crews took the time to obtain the electrocardiogram on scene, or if the "scoop-and-run" method was utilized. To avoid this uncertainty, standardization of the "scoop-and-run" method is being implemented through further education and training of emergency medical services crews responding to STEMI cases. With regard to certain Emergency Department and Transfers patients where the initial electrocardiogram is non-diagnostic, the utilized electrocardiogram-to-device time is inherently shorter than the standard DTD time, since it does not include the time period from arrival to obtaining the electrocardiogram. Nonetheless, it is a necessary surrogate in these cases, since the notification protocol for a STEMI case is not initiated until a diagnostic electrocardiogram is obtained. Concerning transfers, there is no differentiation between patients arriving by personal vehicle to an outside emergency department and those being brought by prehospital ambulance. More uniformity between measurements of DTD time will come once the FTD time is adopted as a performance indicator for all methods of arrival as per the 2013 ACC/AHA guidelines. ${ }^{1}$

A few important findings were identified following initiation of the Rescue One program. The majority of patients arriving by emergency department were able to achieve the goal DTD time of 90 minutes; however there were fewer that were able to do so during OFF hours because of the delay in calling in the catheterization team from home. Methods are being implemented to refine the notification protocol for STEMI cases, with efforts focused on shortening the time to assemble the catheterization lab team during OFF hours, transporting the patient to the catheterization lab before all team members arrive, prepping the site for vascular access sooner, and improving the lines of communication between members of the team before arrival to the hospital. Conversely, the majority of transferred patients were unable to achieve the goal D1TD time of 90 minutes, which can be attributed in part to prolonged DIDO times. Ongoing efforts to shorten DTD times have led to improvement in performance over time. Distance from referring hospitals is also a limitation to improving D1TD times for transferred patients, a challenge that is unique to rural tertiary care centers. Better performance is likely to be seen with the more attainable FTD time goal of 120 minutes for this cohort once it is adopted at our institution for national reporting. In cases where the sum of the prehospital ambulance transportation time and the DIDO time exceeds 60 minutes, fibrinolytic therapy should be considered in the absence of contraindications. Median DTD time and 30-day mortality at our institution were also found to be comparable to published data from other rural tertiary care institutions. Finally, seasonal variability was detected in the Field group mainly due to differences in pre-hospital ambulance transportation times but was not a factor in achieving goal DTD times. Future studies taking into account ambulance transportation times to referring facilities may be warranted.

\section{Acknowledgements}

Great thanks to Julie Uebel from the St. Joseph's Hospital Heart Care Registry for providing the DTD time and in-house mortality data, and to Crystal Jacobson for obtaining the 30-day mortality data. We also thank Dr. Ingrid Glurich and Marie Fleisner for editing and formatting the manuscript, tables, and figures. Finally, we would like to thank Dr. Po-Huang Chyou for providing guidance on the biostatistics performed in this project.

\section{References}

1. American College of Emergency Physicians; Society for Cardiovascular Angiography and Interventions, O'Gara PT, Kushner FG, Ascheim DD, Casey DE Jr, Chung MK, de Lemos JA, Ettinger SM, Fang JC, Fesmire FM, Franklin BA, Granger CB, Krumholz HM, Linderbaum JA, Morrow DA, Newby LK, Ornato JP, Ou N, Radford MJ, Tamis-Holland JE, Tommaso CL, Tracy CM, Woo YJ, Zhao DX, Anderson JL, Jacobs AK, Halperin JL, Albert NM, Brindis RG, Creager MA, DeMets D, Guyton RA, Hochman JS, Kovacs RJ, Kushner FG, Ohman EM, Stevenson WG, Yancy CW. 2013 ACCF/AHA Guideline for the Management of ST-Elevation Myocardial Infarction: A Report of the American College of Cardiology Foundation/American Heart Association Task Force on Practice Guidelines. J Am Coll Cardiol 2013;61:e78-e140.

2. Department of Health and Human Services. Rural Referral Center Program: Rural Health Fact Sheet Series. Centers for Medicare \& Medicaid Services. ICN 006742 January, 2013. Available at: http://www.cms.gov/Outreach-and-Education/ Medicare-Learning-Network-MLN/MLNProducts/ downloads/Rural_Referral_Center_Fact_Sheet.pdf. Accessed May 29, 2013.

3. Shavelle DM, Rasouli ML, Frederick P, Gibson CM, French WJ; National Registry of Myocardial Infarction Investigators. Outcome in patients transferred for percutaneous coronary intervention (a national registry of myocardial infarction 2/3/4 analysis). Am J Cardiol 2005;96:1227-1232.

4. Niles NW, Conley SM, Yang RC, Vanichakarn P, Anderson TA, Butterly JR, Robb JF, Jayne JE, Yanofsky NN, Proehl JA, Guadagni DF, Brown JR; Dartmouth-Hitchcock ST Elevation Myocardial Infarction Process Upgrade Project (STEPUP) Group. Primary percutaneous coronary intervention for patients presenting with ST-segment elevation myocardial infarction: process improvement in a rural ST-segment elevation myocardial infarction receiving center. Prog Cardiovasc Dis 2010;53:202-209. 
5. Blankenship JC, Scott TD, Skelding KA, Haldis TA, TompkinsWeber K, Sledgen MY, Donegan MA, Buckley JW, Sartorius JA, Hodgson JM, Berger PB. Door-to-balloon times under 90 min can be routinely achieved for patients transferred for ST-segment elevation myocardial infarction percutaneous coronary intervention in a rural setting. J Am Coll Cardiol 2011;57:272-279.

6. Rasmussen DK, Washington A, Dougherty J, Fetcko L. Door-toballoon time for primary percutaneous coronary intervention: how does Northern West Virginia compare? J Emerg Med 2012;43:413-416.

7. Sorita A, Ahmed A, Starr SR, Thompson KM, Reed DA, Prokop L, Shah ND, Murad MH, Ting HH. Off-hour presentation and outcomes in patients with acute myocardial infarction: systematic review and meta-analysis. BMJ 2014;348:f7393.

8. Wang TY, Nallamothu BK, Krumholz HM, Li S, Roe MT, Jollis JG, Jacobs AK, Holmes DR, Peterson ED, Ting HH. Association of door-in to door-out time with reperfusion delays and outcomes among patients transferred for primary percutaneous coronary intervention. JAMA 2011;305: 2540-2547.

9. Aguirre FV, Varghese JJ, Kelley MP, Lam W, Lucore CL, Gill JB, Page L, Turner L, Davis C, Mikell FL; Stat Heart Investigators. Rural interhospital transfer of ST-elevation myocardial infarction patients for percutaneous coronary revascularization: the Stat Heart Program. Circulation 2008; 117:1145-1152.

10. Menees DS, Peterson ED, Wang Y, Curtis JP, Messenger JC, Rumsfeld JS, Gurm HS. Door-to-balloon time and mortality among patients undergoing primary PCI. N Engl J Med 2013:5;369:901-909.

\section{Author Affiliations}

Victor A. Abrich, $M D^{*, 1}$; Roxann Rokey, $M D^{*}$; Satya S.V.

Bhupathi, MD, MPH* ; Juan E. Mesa, MD'

${ }^{*}$ Department of Internal Medicine, Marshfield Clinic, Marshfield, Wisconsin, USA

'Department of Cardiology, Marshfield Clinic, Marshfield, Wisconsin, USA

${ }^{1}$ Current Affiliation: Department of Cardiovascular Disease, Mayo Clinic Arizona, Scottsdale, Arizona, USA 